TRANSACTIONS OF THE

AMERICAN MATHEMATICAL SOCIETY

Volume 181, July 1973

\title{
GENERALIZED LAPLACIANS \\ AND MULTIPLE TRIGONOMETRIC SERIES
}

BY

\author{
M. J. KOHN
}

ABSTRACT. V. L. Shapiro gave a $k$-variable analogue for Riemann's theorem on formal integration of trigonometric series. This paper derives Shapiro's results with weaker conditions on the coefficients of the series and extends the results to series which are Bochner-Riesz summable of larger order.

1. Introduction. Let $f(x), x=\left(x_{1}, \cdots, x_{k}\right)$, be a complex valued function defined in a neighborhood of $x_{0} \in E^{k}, k \geq 2$. We will say $f(x)$ has at $x_{0}$ a generalized $r$ th Laplacian equal to $s$ if $f(x)$ is integrable over each sphere $\left|x-x_{0}\right|=t$, for $t$ small, and if

$$
(2 \pi)^{-k / 2} \int_{|y|=1} f\left(x_{0}+t y\right) d s(y)=a_{0}+a_{2} t^{2}+\cdots+a_{2 r} t^{2 r}+o\left(t^{2 r}\right)
$$

as $t \rightarrow 0$, where $a_{2 r}=s / 2^{k / 2+2 r-1} r ! \Gamma(r+k / 2)$. This definition is due to Shapiro [4], who showed that if all partial derivatives of $f$ of order $2 r$ exist and are continuous in a neighborhood of $x_{0}$, then the generalized $r$ th Laplacian of $f\left(x_{0}\right)$ exists and equals $\Delta^{r} f\left(x_{0}\right)$.

Let

$$
T: \sum c_{n} e^{i n \cdot x}
$$

be a trigonometric series in $k$ variables, with $n=\left(n_{1}, \cdots, n_{k}\right), x=\left(x_{1}, \cdots, x_{k}\right)$, $n \cdot x=n_{1} x_{1}+\cdots+n_{k} x_{k}$, and $|n|=\left(n_{1}^{2}+\cdots+n_{k}^{2}\right)^{1 / 2}$. For $\alpha \geq 0$ we denote by

$$
\sigma_{R}^{a}(x)=\sum_{|n|<R}\left(1-\left(\frac{|n|}{R}\right)^{2}\right)^{a} c_{n} e^{i n \cdot x}
$$

the (spherical) Bochner-Riesz means of order $\alpha$ of (1.1). We say $T$ is summable $(B R, \alpha)$ at $x$ to sum $s$ if $\lim _{R \rightarrow \infty} \sigma_{R}^{a}(x)=s$.

Shapiro [4] proved the following result, which is a $k$-dimensional analogue of Riemann's theorem on twice integrated trigonometric series (see, for example, [5, Vol. I, p. 319]).

Suppose $T: \Sigma_{c_{n}} e^{i n \cdot x}$ is $(B R, m)$ summable at $x_{0}$ to the finite sum $s$,

Received by the editors December 20, 1971 and, in revised form, September 11, 1972.

AMS (MOS) subject classifications (1970). Primary 42A24, 42A48, 42A92.

Key words and phrases. Multiple trigonometric series, generalized Laplacian, Bochner-Riesz summable. 
where $m$ is an integer $\geq 0$, and where $c_{n}=O\left(|n|^{\alpha}\right)$, for some $a<m+2-k$, as $|n| \rightarrow \infty$. Let $r$ be an integer $\geq m / 2+1$. Set

$$
F(x)=\frac{c_{0}\left(x_{1}+\cdots+x_{k}\right)^{2 r}}{k^{r}[(2 r) !]}+\sum_{|n| \neq 0} \frac{(-1)^{r} c_{n}}{|n|^{2 r}} e^{i n \cdot x} .
$$

Then the generalized rth Laplacian of $F(x)$ exists at $x_{0}$ and is equal to $s$.

The purpose of this paper is to prove the conclusion of Shapiro's theorem under weaker hypotheses than those stated above. We are able to reduce the hypothesis on the decay of the coefficients of (1.1) and also on the order of summability required. Our result is

Theorem. Let the series (1.1) be summable $(B R, \beta)$ at $x_{0}$ to a finite sum $s$, where $\beta$ is a real number $\geq 0$. Let $r$ be an integer such that $r>1 / 2(\beta+1)$, and suppose $\Sigma|n|^{\gamma}\left|c_{n}\right|^{2}<\infty$ for some $\gamma>k-1-4 r$. Then $F(x)$ defined by

$$
F(x)=\frac{c_{0}\left(x_{1}+\cdots+x_{k}\right)^{2 r}}{k^{r}[(2 r) !]}+\lim _{R \rightarrow \infty} \sum_{0<|n|<R}(-1)^{r} \frac{c_{n}}{|n|^{2 r}} e^{i n \cdot x}
$$

has a generalized $r$ th Laplacian at $x_{0}$ equal to sum $s$.

We note, in particular, that our reduced condition on the growth of the coefficients $c_{n}$ no longer implies that the series defining $F(x)$ converges uniformly. Hence our improvement extends Shapiro's result to the case when $F(x)$ is not necessarily continuous.

2. Before beginning the proof of the Theorem, we note that the Theorem is true if $T$ is only a constant term; see, for example, [2, p. 289]. We may therefore assume $c_{0}=0$. We will also assume, as we may, that $x_{0}=0, s=0$, and that $r$ is the smallest integer with $2 r>\beta+1$. Write $\beta=m+\alpha$ where $m$ is an integer and $0 \leq a<1$.

Write $S_{R}=S_{R}(0)=\Sigma_{|n|<R}{ }_{n}$ and for $\beta>0$ put

$$
S_{R}^{\beta}=\frac{1}{\Gamma(\beta)} \int_{0}^{R}(R-u)^{\beta-1} S_{u} d u .
$$

$S_{R}^{\beta}$, as a function of $R$, is the fractional integral of order $\beta$ of $f(R)=S_{R}$. Hardy has shown that $\sigma_{R}^{\beta}(x) \rightarrow s$ if and only if $\bar{\sigma}_{R}^{\beta}(x) \rightarrow s$, where

$$
\bar{\sigma}_{R}^{\beta}(x)=\frac{\beta}{R^{\beta}} \int_{0}^{R} S_{u}(x)(R-u)^{\beta-1} d u .
$$

Thus the series $(1.1)$ is $(B R, \beta)$ summable to zero at $x=0$ if and only if

$$
S_{R}^{\beta}=o\left(R^{\beta}\right) \text {, }
$$

as $R \rightarrow \infty$. 
3. We begin with some lemmas. Lemmas 1 and 2 are modifications of lemmas from [4].

Lemma 1. Assume $\Sigma|n|^{\gamma}\left|c_{n}\right|^{2}<\infty$ for some $\gamma>k-4 r-1$ and suppose $\Sigma_{c_{n}}$ is $(B R, m+1)$ summable to zero. Then $S_{R}^{n}=o\left(R^{2 r+1 / 2}\right)$ for $n=0, \cdots, m$, as $R \rightarrow \infty$.

Proof. Write $\gamma=k-4 r-1+\epsilon$, where $\epsilon>0$. Then

$$
\begin{aligned}
\sum_{|n|<R}\left|c_{n}\right| & =\sum_{|n|<R}|n|^{\gamma / 2}\left|c_{n}\right||n|^{-\gamma / 2} \leq\left(\sum|n|^{\gamma}\left|c_{n}\right|^{2}\right)^{1 / 2}\left(\sum_{|n|<R}|n|^{-\gamma}\right)^{1 / 2} \\
& =C\left(R^{-\gamma+k}\right)^{1 / 2}=C R^{1 / 2(-k+4 r+1-\epsilon+k)}=o\left(R^{2 r+1 / 2}\right) .
\end{aligned}
$$

Note that for $j=1,2, \cdots, m+2$,

$$
\begin{gathered}
\sum_{|s|<R} c_{s}(R-|s|+j)^{m+1}=\sum_{|s|<R+j}-\sum_{R \leq|s|<R+j}=\mathrm{I}+\mathrm{II} . \\
\mathrm{I}=o\left(R^{m+1}\right) \text { since } \sum_{c_{s}} \text { is }(B R, m+1) \text { summable to } 0 . \\
|\mathrm{II}| \leq C \sum_{|s|<R+j}\left|c_{s}\right|=o\left(R^{2 r+1 / 2}\right), \text { by }(3.1) .
\end{gathered}
$$

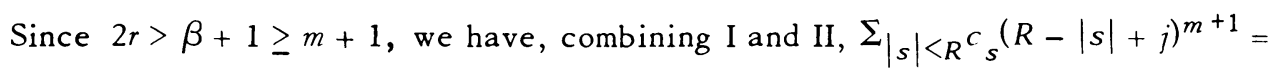
$o\left(R^{2 r+1 / 2}\right)$.

We observe that there exist numbers $A_{j n}$ for $j=1, \cdots, m+2$ and $n=0, \cdots, m$ such that $\sum_{j=1}^{m+2} A_{j n}(z+j)^{m+1}=z^{n}$ for $z \in \mathbf{C}$. This is Lemma 3 of [4]. Hence

$$
\begin{aligned}
S_{R}^{n} & =\frac{1}{n !} \sum_{|s|<R} c_{s}\left(R-|s|^{n}\right)=\frac{1}{n !} \sum_{|s|<R} c_{s} \sum_{j=1}^{m+2} A_{j n}(R-|s|+j)^{m+1} \\
& =\sum_{j=1}^{m+2} \frac{1}{n !} A_{j n} \sum_{|s|<R} c_{s}(R-|s|+j)^{m+1}=\sum_{j=1}^{m+2} \frac{1}{n !} A_{j n} o\left(R^{2 r+1 / 2}\right)=o\left(R^{2 r+1 / 2}\right) .
\end{aligned}
$$

Lemma 2. Let $J_{s}(t)$ denote the Bessel function of the first kind of order $s$, where $s$ is an integer or balf integer. Then for each $n>0$,

$$
\frac{d^{n}}{d t^{n}} \frac{J_{s}(t)}{t^{s+2 r}}=O\left(t^{-s-2 r-1 / 2}\right),
$$

as $t$ tends to infinity.

Proof. The argument is the same as the proof of Lemma 2 of [4]. However we use here the fact that $J_{s}(t)=O\left(t^{-1 / 2}\right)$ as $t \rightarrow \infty$.

Lemma 3. If $0 \leq a<1$, then $\int_{0}^{u}(u-z)^{-\alpha} z^{\alpha} d z=O(u)$ as $u$ tends to infinity. 
Proof.

$$
\int_{0}^{u}(u-z)^{-\alpha} z^{\alpha} d z=\int_{0}^{u / 2}+\int_{u / 2}^{u}=A+B
$$

where

$$
\begin{aligned}
& A=\int_{0}^{u / 2}(u-z)^{-a} z^{a} d z=O\left(u^{-a}\right) \int_{0}^{u / 2} z^{a} d z=O(u), \\
& B=\int_{u / 2}^{u}(u-z)^{-a} z^{\alpha} d z=O\left(u^{\alpha}\right) \int_{u / 2}^{u}(u-z)^{-a} d z=O\left(u^{\alpha}\right) O\left(u^{1-a}\right)=O(u) .
\end{aligned}
$$

Lemma 4. With $S_{u}^{\beta}$ defined by (2.1), then for almost all $u$

Proof. Put

$$
S_{u}^{m}=\frac{1}{\Gamma(1-\alpha)} \frac{d}{d u} \int_{0}^{u}(u-z)^{-\alpha} S_{z}^{\alpha+m} d z
$$

$$
I=\frac{1}{\Gamma(1-\alpha)} \int_{0}^{u}(u-z)^{-\alpha} S_{z}^{a+m} d z=\frac{1}{\Gamma(1-\alpha)} \int_{0}^{u}(u-z)^{(1-\alpha)-1} S_{z}^{a+m} d z .
$$

Thus $I$ is the fractional integral of order $(1-\alpha)$ of $S_{u}^{a+m}$. But since $S_{u}^{\alpha+m}$ is the fractional integral of order $(\alpha+m)$ of $S_{u}$, therefore $I$ is the fractional integral of $S_{u}$ of order $(\alpha+m)+(1-\alpha)=m+1$. Hence for almost all $u, d I / d u=$ $S_{u}^{m}$

4. Proof of the Theorem. We must show

$$
(2 \pi)^{-k / 2} \int_{z \in \mathbf{\Sigma}} F(t z) d s(z)=a_{0}+a_{2} t^{2}+\cdots+a_{2(r-1)} t^{2(r-1)}+o\left(t^{2 r}\right)
$$

as $t$ tends to zero.

The hypothesis on the moduli of $c_{n}$ implies $\left.\left.\Sigma|n|^{k-1+\epsilon}\left|c_{n}\right| n\right|^{-2 r}\right|^{2}<\infty$, for some $\epsilon>0$. Therefore by virtue of Theorem 1 of [3] the series defining $F(x)$ in (1.3) converges spherically almost everywhere on each sphere $|x|=t$. By virtue of Theorem 2 of [3], with $p=1$, we may integrate this series term by term over each sphere.

Bochner [1] observed $(2 \pi)^{-k / 2} \int_{z \in \Sigma^{2}} e^{i n t \cdot z} d s(z)=J_{s}(|n| t) /(|n| t)^{s}$, where $s=$ $1 / 2(k-2)$. Hence,

$$
\begin{aligned}
(2 \pi)^{-k / 2} \int_{\Sigma} F(t z) d s(z) & =\lim _{R \rightarrow \infty} \sum_{0<|n|<R}(-1)^{r} \frac{c_{n}}{|n|^{2 r}}(2 \pi)^{-k / 2} \int_{\Sigma} e^{i n \cdot t z} d s(z) \\
& =\lim _{R \rightarrow \infty}(-1)^{r} \sum_{0<|n|<R} \frac{c_{n}}{|n|^{\frac{2 r}{r}}} \frac{J_{s}(|n| t)}{\left(\mid n_{i} t^{s}\right.}=(-1)^{r} t^{2 r} \lim _{R \rightarrow \infty} \sum_{0<|n|<R} c_{n} \gamma(|n| t),
\end{aligned}
$$

where $\gamma(u)=u^{-s-2 r} J_{s}(u)$.

We apply summation by parts to $\Sigma_{|n|<R}{ }_{n} \gamma(|n| t) m$ times. 


$$
\begin{aligned}
\sum_{|n|<R} c_{n} \gamma(|n| t) & =S_{R} \gamma(R t)-\int_{0}^{R} S_{u} \frac{d}{d u} \gamma(u t) d u \\
= & S_{R} \gamma(R t)-S_{R}^{1} \frac{d}{d R} \gamma(R t)+\int_{0}^{R} S_{u}^{1} \frac{d^{2}}{d u} \gamma(u t) d u \\
& \vdots \\
= & S_{R} \gamma(R t)-S_{R}^{1} \frac{d}{d R} \gamma(R t)+\cdots+(-1)^{m} S_{R}^{m} \frac{d^{m}}{d R^{m}} \gamma(R t) \\
& +(-1)^{m+1} \int_{0}^{R} S_{u}^{m} \frac{d^{m+1}}{d u^{m+1}} \gamma(u t) d u .
\end{aligned}
$$

Using Lemmas 1 and 2 , with $j=1, \cdots, m, S_{R}^{j}\left(d^{j} / d R^{j}\right) \gamma(R t)=o\left(R^{2 r+1 / 2}\right)$. $O\left(R^{-(k-2) / 2-2 r-1 / 2}\right)=O(1)$, as $R$ tends to infinity. Thus,

$$
\lim _{R \rightarrow \infty} \sum_{0<i n \mid<R} c_{n} \gamma(|n| t)=(-1)^{m+1} \int_{0}^{\infty} S_{u}^{m} \frac{d^{m+1}}{d u^{m+1}} \gamma(u t) d u,
$$

and returning to $(4.1)$ we get

$$
\begin{aligned}
(2 \pi)^{-k / 2} \int_{\Sigma} F(t z) d s(z) & =(-1)^{r} t^{2 r} \sum_{0<|n|<R} c_{n} \gamma(|n| t) \\
& =(-1)^{r+m+1} t^{2 r} \int_{1}^{\infty} S_{u}^{m} \frac{d^{m+1}}{d u^{m+1}} \gamma(u t) d u .
\end{aligned}
$$

We use the formula $J_{s}(z)=\sum_{n=0}^{\infty}\left((-1)^{n}(1 / 2 z)^{s+2 n} / n ! \Gamma(s+n+1)\right)$. Hence

$$
\begin{aligned}
z^{-s} J_{s}(z) & =\sum_{n=0}^{\infty} \frac{(-1)^{n}(2)^{-s-2 n} z^{2 n}}{n ! \Gamma(s+n+1)} \\
& =c_{0}+c_{2} z^{2}+\cdots+c_{2 n} z^{2 n}+\cdots
\end{aligned}
$$

Define $P(z)=c_{0}+c_{2} z^{2}+\cdots+c_{2(r-1)} z^{2(r-1)}$ and let $\lambda(z)=\left(z^{-s} J_{s}(z)-P(z)\right) / z^{2 r}$. Then $\lambda(z)$ is an entire function in the plane and

$$
\gamma(z)=\lambda(z)+z^{-2 r} P(z) .
$$

Substituting into (4.2),

$$
(2 \pi)^{-k / 2} \int_{\Sigma} F(t z) d s(z)=(-1)^{r+m+1} t^{2 r} \int_{1}^{\infty} S_{u}^{m} \frac{d^{m+1}}{d u^{m+1}}\left[\frac{P(u t)}{(u t)^{2 r}}+\lambda(u t)\right] d u
$$

$$
\begin{aligned}
& =(-1)^{r+m+1} \int_{1}^{\infty} S_{u}^{m} \frac{d^{m+1}}{d u^{m+1}} \frac{P(u t)}{u^{2 r}} d u+(-1)^{r+m+1} t^{2 r} \int_{1}^{\infty} S_{u}^{m} \frac{d^{m+1}}{d u^{m+1}} \lambda(u t) d u \\
& =A(t)+t^{2 r} B(t) .
\end{aligned}
$$




$$
\begin{aligned}
A(t) & =(-1)^{r+m+1} \int_{1}^{\infty} S_{u}^{m} \frac{d^{m+1}}{d u^{m+1}} \frac{P(u t)}{u^{2 r}} d u \\
& =(-1)^{r+m+1} \int_{1}^{\infty} S_{u}^{m} \frac{d^{m+1}}{d u^{m+1}}\left\{\sum_{j=0}^{r-1} c_{j} \frac{(u t)^{2 j}}{u^{2 r}}\right\} d u \\
& =(-1)^{r+m+1} \sum_{j=0}^{r-1} t^{2 j} \int_{1}^{\infty} S_{u}^{m} \frac{d^{m+1}}{d u^{m+1}} c_{j} u^{2(j-r)} d u=\sum_{j=0}^{r-1} A_{2 j} t^{2 j} .
\end{aligned}
$$

We will show $A_{2 j}$ is finite for each $j$.

$$
A_{2 j}=c_{j}^{\prime} \int_{0}^{\infty} S_{u}^{m} u^{2(j-r)-m-1} d u=c_{j}^{\prime} \int_{1}^{\infty} S_{u}^{m} u^{-n_{j}} d u,
$$

with $m+3 \leq n_{j} \leq 2 r+m+1$. By Lemma 4,

$$
S_{u}^{m}=(1 / \Gamma(1-\alpha))(d / d u) \int_{0}^{u}(u-z)^{-\alpha} S_{z}^{a+m} d z,
$$

for almost all $u$. Hence

$$
A_{2 j}=c_{j}^{\prime \prime} \int_{1}^{\infty} u^{-n_{j}} \frac{d}{d u} \int_{0}^{u}(u-z)^{-\alpha} S_{z}^{\alpha+m} d z d u .
$$

Integrating by parts,

$$
A_{2 j}=\left.c_{j}^{\prime \prime} u^{-n_{j}} \int_{0}^{u}(u-z)^{-\alpha} S_{z}^{\alpha+m} d z\right|_{1} ^{\infty}-c_{j}^{\prime \prime} \int_{1}^{\infty} u^{-n_{j}-1} \int_{0}^{u}(u-z)^{-\alpha_{2}^{\alpha+m}} d z d u=\alpha_{j}+\beta_{j} .
$$

By (2.2), we have $S_{R}^{a+m}=o\left(R^{\alpha+m}\right)$, so

$$
\begin{aligned}
& \alpha_{j}=\left.c_{j}^{\prime \prime} u^{-n_{j}} \int_{0}^{u}(u-z)^{-\alpha}{ }_{o}\left(z^{\alpha+m}\right) d z\right|_{1} ^{\infty} \\
& =\left.o\left(u^{-n_{j}+m}\right) \int_{0}^{u}(u-\dot{z})^{-a} z^{\alpha} d z\right|_{1} ^{\infty}=\left.o\left(u^{-n_{j}+m}\right) O(u)\right|_{1} ^{\infty}
\end{aligned}
$$

by Lemma 3. Since $n_{j} \geq m+3, \alpha_{j}=O(1)$.

To estimate $\beta_{j}$ we again use Lemma 3 .

$$
\begin{aligned}
\beta_{j} & =c_{j}^{\prime \prime} \int_{1}^{\infty} u^{-n_{j}-1} \int_{0}^{u}(u-z)^{-\alpha_{o}}\left(z^{\alpha+m}\right) d z d u \\
& =c_{j}^{\prime \prime} \int_{1}^{\infty} u^{-n_{j}-1} o\left(u^{m}\right) \int_{0}^{u}(u-z)^{-\alpha_{o}\left(z^{\alpha}\right) d z d u} \\
& =c_{j}^{\prime \prime} \int_{1}^{\infty} o\left(u^{-n_{j}-1+m}\right) O(u) d u=O(1) .
\end{aligned}
$$

This shows that each $A_{2 j}=\alpha_{j}+\beta_{j}$ is finite. Combining this last statement with (4.4) and (4.5), we obtain

$$
(2 \pi)^{-k / 2} \int_{\Sigma} F(t z) d s(z)=\sum_{j=0}^{r-1} A_{2 j} t^{2 j}+t^{2 r} B(t) .
$$

5. The proof of the Theorem will be complete when we show $B(t)$ tends to zero with $t$. Using Lemma 4 , 


$$
\begin{aligned}
B(t) & =(-1)^{r+m+1} \int_{1}^{\infty} S_{u}^{m} \frac{d^{m+1}}{d u^{m+1}} \lambda(u t) d u \\
& =(-1)^{r+m+1} \int_{0}^{\infty} \frac{1}{\Gamma(\alpha+m)} \frac{d}{d u} \int_{0}^{u}(u-z)^{-a_{S} S_{z}^{a+m}} d z \frac{d^{m+1}}{d u^{m+1}} \lambda(u t) d u
\end{aligned}
$$

(We are justified in replacing the limits of integration in the outer integral by $0 \leq$ $u<\infty$ because $S_{u}^{m}=0$ for $0 \leq u<1$.) We integrate the expression for $B(t)$ by parts.

$$
\begin{aligned}
B(t)= & \left.\frac{(-1)^{r+m+1}}{\Gamma(\alpha+m)} \int_{0}^{u}(u-z)^{-a_{S}} \int_{z}^{\alpha+m} d z \cdot \frac{d^{m+1}}{d u^{m+1}} \lambda(u t)\right|_{0} ^{\alpha} \\
& +\frac{(-1)^{r+m}}{\Gamma(\alpha+m)} \int_{0}^{\infty} \int_{0}^{u}(u-z)^{-\alpha_{S} \alpha+m} d z \cdot \frac{d^{m+2}}{d u^{m+2}} \lambda(u t) d u \\
= & B_{1}(t)+B_{2}(t) .
\end{aligned}
$$

We assert that, for $t \neq 0, B_{1}(t)$ is zero. Since $\lambda(z)$ is entire in the plane, $d^{m+1} \lambda(u t) / d u^{m+1}$ remains bounded as $u$ tends to zero. Hence at zero the expression defining $B_{1}(t)$ is zero. To evaluate the expression defining $B_{1}(t)$ at $u=\infty$ we use (4.3) and (2.2).

$$
\int_{0}^{u}(u-z)^{-\alpha} S_{z}^{a+m} d z \cdot \frac{d^{m+1}}{d u^{m+1}} \lambda(u t)=o\left(u^{m}\right) \int_{0}^{u}(u-z)^{-a} z^{a} d z \cdot \frac{d^{m+1}}{d u^{m+1}}\left[\gamma(u t)-\frac{P(u t)}{(u t)^{2 r}}\right] .
$$

The integral in this last expression is $O(u)$ by Lemma 3. By Lemma 2, $d^{m+1} \gamma(u t) / d u^{m+1}=O\left(u^{-m-1}\right)$, and since $P(u t)(u t)^{-2 r}$ is a quotient of polynomials with the degree of the denominator greater by two than the degree of the numerator, $\left(d^{m+1} / d u^{m+1}\right) P(u t) /(u t)^{2 r}=O\left(u^{-2-m-1}\right)$ as $u \rightarrow \infty$. Hence, near $u=\infty$, the expression defining $B_{1}(t)$ is $o\left(u^{m}\right) O(u)\left[O\left(u^{-m-1}\right)+O\left(u^{-m-1}\right)\right]=o(1)$.

It remains to be shown that $B_{2}(t)$ tends to zero with $t$.

$$
\begin{aligned}
B_{2}(t) & =\frac{(-1)^{r+m}}{\Gamma(a+m)} \int_{0}^{\infty} \int_{0}^{u}(u-z)^{-a} S_{z}^{a+m} d z \frac{d^{m+2}}{d u^{m+2}} \lambda(u t) d u \\
& =\frac{(-1)^{r+m}}{\Gamma(\alpha+m)} \int_{0}^{\infty} S_{z}^{a+m}\left\{\int_{z}^{\infty}(u-z)^{-a} \frac{d^{m+2}}{d u^{m+2}} \lambda(u t) d u\right\} d z \\
& =\frac{(-1)^{r+m}}{\Gamma(\alpha+m)} \int_{0}^{\infty} S_{z}^{a+m} H(z, t) d z=\frac{(-1)^{r+m}}{\Gamma(\alpha+m)}\left\{\int_{0}^{1 / t}+\int_{1 / t}^{\infty}\right\}=\frac{(-1)^{r+m}}{\Gamma(a+m)}\{M+N\} .
\end{aligned}
$$

We first consider $M$. In this integral $z$ ranges between 0 and $1 / t$, and

$$
H(z, t)=\int_{z}^{\infty}(u-z)^{-\alpha} \frac{d^{m+2}}{d u^{m+2}} \lambda(u t) d u=\int_{z}^{1 / t}+\int_{1 / t}^{\infty}=P+Q .
$$


$\lambda(z)$ is entire, so for $|u t|<1, d^{m+2} \lambda(u t) / d u^{m+2}=O\left(t^{m+2}\right)$. Hence

$$
\begin{aligned}
P & =\int_{z}^{1 / t}(u-z)^{-\alpha} O\left(t^{m+2}\right) d u=O\left(t^{m+2}\right)(1 / t-z)^{1-\alpha} \\
& =O\left(t^{m+1}\right)(1 / t-z)^{1-a}(t(1 / t-z))=O\left(t^{m+1}\right)(1 / t-z)^{1-\alpha},
\end{aligned}
$$

since $t(1 / t-z)=1-t z \leq 1$.

$$
\begin{aligned}
Q & =\int_{1 / t}^{\infty}(u-z)^{-a} \frac{d^{m+2}}{d u^{m+2}} \lambda(u t) d u \\
& =\int_{1 / t}^{\infty}(u-z)^{-\alpha} \frac{d^{m+2}}{d u^{m+2}} \gamma(u t) d u+\int_{1 / t}^{\infty}(u-z)^{-a} \frac{d^{m+2}}{d u^{m+2}} \frac{P(u t)}{(u t)^{2 r}} d u=Q_{1}+Q_{2} .
\end{aligned}
$$

By Lemma 2,

$$
\frac{d^{m+2}}{d u^{m+2}} \gamma(u t)=t^{m+2} O\left((u t)^{-s-2 r-1 / 2}\right)=t^{m+3 / 2-2 r-s} O\left(u^{-s-2 r-1 / 2}\right) .
$$

Hence,

$$
\begin{aligned}
Q_{1} & =\int_{1 / t}^{\infty}(u-z)^{-a_{t} m+3 / 2-2 r-s} O\left(u^{-s-2 r-1 / 2}\right) d u \\
& =(1 / t-z)^{-a_{t} m+3 / 2-2 r-s} \int_{1 / t}^{\infty} O\left(u^{-s-2 r-1 / 2}\right) d u=(1 / t-z)^{-a} O\left(t^{m+1}\right) .
\end{aligned}
$$

Since $\left(d^{m+2} / d u^{m+2}\right)\left[P(u t)(u t)^{-2 r}\right]=t^{-2} O\left(u^{-m-4}\right)$, therefore

$$
Q_{2}=\int_{1 / t}^{\infty}(u-z)^{-a} t^{-2} O\left(u^{-m-4}\right) d u=(1 / t-z)^{-a} O\left(t^{-2+m+3}\right) .
$$

Combining the estimates for $Q_{1}$ and $Q_{2}$ we get $Q=(1 / t-z)^{-a} O\left(t^{m+1}\right)$, and with (5.1) and (5.2) we conclude, for $0 \leq z \leq 1 / t, H(z, t)=(1 / t-z)^{-a} O\left(t^{m+1}\right)$. Therefore

$$
\begin{aligned}
M & =\int_{0}^{1 / t} S_{z}^{a+m} H(z, t) d z=\int_{0}^{1, t} o\left(z^{\alpha+m}\right) O\left(t^{m+1}\right)(1 / t-z)^{-\alpha} d z \\
& =o(1 / t)^{a+m} t^{m+1} \int_{0}^{1 / t}(1 / t-z)^{-\alpha} d z=o(1) .
\end{aligned}
$$

We now consider $N=\int_{1 / t}^{\infty} S_{z}^{a+m} H(z, t) d z$. In this integral $z$ is larger than $1 / t$.

$$
\begin{aligned}
H(z, t) & =\int_{z}^{\infty}(u-z)^{-a} \frac{d^{m+2}}{d u^{m+2}} \lambda(u t) d u \\
& =\int_{z}^{\infty}(u-z)^{-a} \frac{d^{m+2}}{d u^{m+2}} \gamma(u t) d u+\int_{z}^{\infty}(u-z)^{-a} \frac{d^{m+2}}{d u^{m+2}} \frac{P(u t)}{(u t)^{2 r}} d u=R+S .
\end{aligned}
$$

To compute $R$ we apply Lemma 2 .

$$
\left(d^{m+2} / d u^{m+2}\right) \gamma(u t)=t^{m+3 / 2-s-2 r} O\left(u^{-s-2 r-1 / 2}\right) .
$$

Hence, 


$$
\begin{gathered}
R=t^{m+3 / 2-s-2 r} \int_{z}^{\infty}(u-z)^{-a} O\left(u^{-s-2 r-1 / 2}\right) d u \\
=t^{m+3 / 2-s-2 r}\left[\int_{z}^{2 z}+\int_{2 z}^{\infty}\right] \\
\int_{z}^{2 z}(u-z)^{-a} O\left(u^{-s-2 r-1 / 2}\right) d u=O\left(z^{-s-2 r-1 / 2}\right) \int_{z}^{2 z}(u-z)^{-a} d u \\
=O\left(z^{-s-2 r-1 / 2}\right) O\left(z^{1-\alpha}\right)=O\left(z^{-s-2 r+1 / 2-\alpha}\right), \\
\int_{2 z}^{\infty}(u-z)^{-a} O\left(u^{-s-2 r-1 / 2}\right) d u=O\left(z^{-a}\right) \int_{2 z^{\infty}}^{\infty} u^{-s-2 r-1 / 2} d u \\
=O\left(z^{-a}\right) O\left(z^{-s-2 r+1 / 2}\right)=O\left(z^{-s-2 r+1 / 2-a}\right) .
\end{gathered}
$$

Thus, $R=t^{m+3 / 2-s-2 r} O\left(z^{-s-2 r+1 / 2-a}\right)=t^{-1 / 2} O\left(z^{-a-m-3 / 2}\right)(t z)^{m+2-s-2 r}$. Since $|t z| \geq 1$ in the interval of integration and since $2 r \geq m+2$ and $s \geq 0$, the last factor on the right of this last equation is $\leq 1$. Hence,

$$
R=t^{-1 / 2} O\left(z^{-a-m-3 / 2}\right) \text {. }
$$

To compute $S$, we again use the fact that $\left(d^{m+2} / d u^{m+2}\right) P(u t) /(u t)^{2 r}=$ $t^{-2} O\left(u^{-m-4}\right)$; and therefore

$$
\begin{gathered}
S=\int_{z}^{\infty}(u-z)^{-a} \frac{d^{m+2}}{d u^{m+2}} \frac{P(u t)}{(u t)^{2 r}} d u \\
=t^{-2} \int_{z}^{\infty}(u-z)^{-a} O\left(u^{-m-4}\right) d u=t^{-2}\left\{\int_{z}^{2 z}+\int_{2 z}^{\infty}\right\} \\
\int_{z}^{2 z}(u-z)^{-a} O\left(u^{-m-4}\right) d u=O\left(z^{-m-4}\right) \int_{z}^{2 z}(u-z)^{-a} d u \\
=O\left(z^{-m-4}\right) O\left(z^{1-a}\right)=O\left(z^{-m-a-3}\right) . \\
\int_{2 z}^{\infty}(u-z)^{-a} O\left(u^{-m-4}\right) d u=O\left(z^{-a}\right) \int_{2 z}^{\infty} u^{-m-4} d u=O\left(z^{-a}\right) z^{-m-3} .
\end{gathered}
$$

Thus $S=t^{-2} O\left(z^{-m-a-3}\right)=t^{-1 / 2} O\left(z^{-m-a-3 / 2}\right)$. Combining with (5.3), we see that for $1 / t<z, H(z, t)=R+S=t^{-1 / 2} O\left(z^{-m-a-3 / 2}\right)$.

Substituting into the integral defining $N$,

$$
\begin{aligned}
N & =\int_{1 / t}^{\infty} S_{z}^{a+m} H(z, t) d z=\int_{1 / t}^{\infty} o\left(z^{a+m}\right) t^{-1 / 2} O\left(z^{-m-a-3 / 2}\right) d z \\
& =t^{-1 / 2} \int_{1 / t}^{\infty} o\left(z^{-3 / 2}\right) d z=t^{-1 / 2} o(1 / t)^{-1 / 2}=o(1) .
\end{aligned}
$$

We have shown that $B_{2}(t)$ tends to zero with $t$. This completes the proof of the Theorem. 


\section{BIBLIOGRAPHY}

1. S. Bochner, Summation of multiple Fourier series by spherical means, Trans. Amer. Math. Soc. 40 (1936), 175-207.

2. R. Courant, Me thods of mathematical physics. Vol. II: Partial differential equations, Interscience, New York, 1962. MR $25 \# 4216$.

3. M. Kohn, Spherical convergence and in te grability of multiple trigonometric series on hypersurfaces, Studia Math. 44 (1972), 345-354.

4. V. L. Shapiro, Circular summability $C$ of double trigonometric series, Trans. Amer. Math. Soc. 76 (1954), 223-233. MR 15, 866.

5. A. Zygmund, Trigonometric series. Vols. 1, 2, 2nd ed., Cambridge Univ. Press, New York, 1968. MR 38 \#4882.

DEPARTMENT OF MATHEMATICS, UNIVERSITY OF MINNESOTA, MINNEAPOLIS, MINNESOTA 55455 